\title{
Impact and Control of Drug Therapy Guidelines for Tumor Patients During the Novel Coronavirus Pneumonia Epidemic
}

\author{
${ }^{1}$ Razi Ahmed, ${ }^{1}$ Shafiza Mohd Shariff, ${ }^{1}$ Shahrinaz Ismail ${ }^{3}$ Anwer Irshad Burney and ${ }^{2}$ Nawaf Waqas \\ ${ }^{1}$ Universiti Kuala Lumpur, Malaysian Institute of Information Technology, Kuala Lumpur, Malaysia \\ ${ }^{2}$ Universiti Kuala Lumpur, British Malaysian Institute, Kuala Lumpur, Malaysia \\ ${ }^{3}$ The Millennium University, Karachi, Pakistan
}

\section{Article history}

Received: 14-03-2021

Revised: 17-05-2021

Accepted: 31-05-2021

Corresponding Author:

Razi Ahmed

Universiti Kuala Lumpur,

Malaysian Institute of

Information Technology, Kuala

Lumpur, Malaysia

Email: razi.ahmed@s.unikl.edu.my

\begin{abstract}
Since December 2019, many unexplained viral pneumonia cases have been found in Wuhan City, Hubei Province, China. It was later confirmed that the outbreak's causative agent was a new coronavirus. The virus was temporarily named "2019-new coronavirus" (2019-nCoV) by the World Health Organization (WHO). The diseases caused by 2019-nCoV were called by the National Health and Health Commission of China "New coronavirus pneumonia" (Novel Coronavirus Pneumonia, NCP) and was named "Coronavirus disease 2019" (COVID-19) by the WHO. The outbreak of NCP seriously affected the lives of the public. This article focuses on the group of cancer patients, comprehensively considers the social, medical resources and family issues to analyze the possible impact of the epidemic on cancer patients' drug treatment and health and makes recommendations for cancer patients' management.
\end{abstract}

Keywords: COVID-19, Cancer, Pneumonia and Healthcare

\section{Introduction}

\section{A. The Possible Impact of NCP Epidemic on Cancer Patients}

\section{a. Risk of NCP in Cancer patients}

Although the population is generally susceptible to 2019-nCoV, it is the elderly and people with underlying diseases who are more seriously affected by this NCP outbreak. The median age of patients with NCP was 47 (35-58) years old, according to the findings of a study of clinical characteristics of 1099 patients with NCP reported on the academic website Med Rxiv by Zhong Nanshan's research team. The median age of severe patients was 52 (40-65). The median age of patients who reached the composite endpoint (admission to the intensive care unit, use of artificial respirators, or death) was 63 (53 to 71) years old and only $0.9 \%$ were under 15 years old. The elderly and patients with underlying diseases such as malignant tumours, diabetes and heart disease often have severe clinical manifestations such as pneumonia and dyspnea and even life-threatening (Huang et al., 2020; Favre et al., 2020; Song et al., 2020; Liang et al., 2020). A study by Liang et al. (2020) showed that 18 of the 1,590 NCP patients $(1.13,95 \%$ CI: 0.61 to 1.65 ) were cancer patients, which was higher than the proportion of cancer patients in the general population of China $(0.29 \%, 285.83$ People/100,000).
Tumour patients have weakened immune function and weak resistance to viral infection. During chemotherapy and radiotherapy treatment, the immune function may be further damaged (Van Helvoort, 2014). In recent years, immune checkpoint inhibitors have developed rapidly in cancer treatment. Still, the occurrence of immunogenic adverse reactions can also increase the risk of viral infection to a certain extent (Moslehi et al., 2018; Johnson et al., 2016). In summary, under the current NCP epidemic environment, cancer patients have a higher risk of 2019-nCoV disease and it is imperative to strengthen protection for cancer patients, a high-risk group.

\section{b. The Effect of the NCP Epidemic on Cancer Patients' Care and Treatment}

The epidemic situation of infectious diseases affects infected patients themselves and patients' health with non-infectious conditions. Taking the Severe Acute Respiratory Syndrome (SARS) epidemic in 2003 as an example, the number of outpatient consultations in the otolaryngology department of a tertiary hospital in Hong Kong decreased by 59\% from March to April 2003. The amount of surgery decreased by $79 \%$, on average, the bed occupancy rate decreased by $79 \%$ and the daily hospitalization rate decreased by $84 \%$ (Vlantis et al., 2004). A survey conducted by the Department of Radiation Oncology at the University of Toronto showed that the peak 
of the SARS epidemic from March 20 to May 30, 2003, compared with the same period in 2002, the number of consultations for cancer patients receiving palliative radiotherapy decreased by $21 \%$. The number of outpatients decreased by $15 \%$ (Raber-Durlacher et al., 2004). The main reason for the decline in the number of noncommunicable disease clinics, operations and treatments in hospitals is the patients' worry about the prevalence of infectious diseases. Taipei Veterans General Hospital surveyed 79 patients with non-small cell lung cancer undergoing chemotherapy during the SARS epidemic. $63.8 \%(37 / 58)$ of patients were afraid of going to the hospital because of the epidemic; $50 \%$ (29/58) answered If an outbreak occurs, you will refuse to go to the hospital for chemotherapy; $36.2 \%(21 / 58)$ of patients think SARS is more severe and fatal than lung cancer; $79.3 \%$ (46/58) of patients worry that the outbreak of SARS will worsen your cancer. During the investigation, 79 of the 373 scheduled chemotherapy patients, $10(2.7 \%)$ were delayed due to the epidemic and $3(3.8 \%)$ patients refused to receive chemotherapy because of fear of getting SARS when going to the hospital (Chen et al., 2004). It is currently in a critical period of NCP epidemic control in China. The focus of medical care is on preventing and controlling the epidemic. Medical personnel from many provinces and cities support Wuhan, Hubei, or directly participating in epidemic prevention and control. Some medical personnel is infected with 2019-nCoV. Facilities and medicines are used for the prevention and treatment of NCP. Some hospital wards are used to isolate patients or quarantine them. Some hospitals' departments are closed due to nosocomial infections, which results in a shortage of medical personnel and resources, which affects the standard treatment of cancer patients treatment. The transportation system has also successfully introduced measures to increase traffic control in the main urban area, restricting people's movement and bringing difficulties to cancer patients' treatment.

The treatment of cancer patients is different from other diseases. Once delayed, it may be due to tumour resistance or distant metastasis. The patient may no longer have the possibility of a clinical cure, shortening the patient's survival time, increasing the pain caused by the patient and even Cause death. Therefore, medical treatment and cancer patients' treatment during the NCP epidemic is critical.

\section{B. The Principle of Anti-Tumor Drug Treatment}

\section{a. The Implementation of "Long Prescription" Policy}

During the epidemic situation, the National Medical Security Administration implemented a "long prescription" reimbursement policy to support medical institutions to increase the number of single prescription drugs reasonably according to patients' actual situation and reduce the number of patients going to medical institutions. For cancer patients with hypertension, diabetes and other chronic diseases, after evaluation by the doctors in the hospital, they support the relaxation of prescription drugs to 3 months to ensure their long-term medication needs (SMSB, 2020). However, it is recorded that cancer patient's medical treatment is still different from other chronic diseases. The treatment process requires regular evaluation of its efficacy and safety, so "long prescription" requires more clinicians. Pharmacists' participation improves the treatment effect and reduces the risk of drug resistance and adverse reactions.

\section{b. The Principles of Administration Delayed/missed Treatment}

During the epidemic, when receiving anti-tumor targeted drug treatment, tumor patients should not take medicine on time in case of emergencies, delays, or misuse. They should contact the clinical pharmacist or clinician of the local hospital's oncology department in time and follow their instructions Suggest. Taking trastuzumab as an example, if patients missed trastuzumab for less than one week, they should be given a conventional maintenance dose of trastuzumab as soon as possible (dose once a week: $2 \mathrm{mg} / \mathrm{kg}$; The dosing regimen every three weeks: 6 $\mathrm{mg} / \mathrm{kg}$ ), no need to wait until the next treatment cycle. If the patient has missed trastuzumab for more than one week, the initial loading dose of trastuzumab should be re-administered as soon as possible (dosing schedule once a week: $4 \mathrm{mg} / \mathrm{kg}$; dosing schedule once every three weeks: $8 \mathrm{mg} / \mathrm{kg}$ ), the infusion time is more than $90 \mathrm{~min}$. After that, a maintenance dose of trastuzumab should be given after seven days or 21 days for the dosing schedule once a week or once every three weeks, respectively (SMSB, 2020).

\section{c. The Infected Cancer Patients with nCoV-2019}

Once cancer patients are infected with 2019-nCoV, they should be treated mainly with $2019-n C o V$ infection. For the 2019-nCoV disease, there are currently no specific drugs. According to China's "New Coronavirus Pneumonia Diagnosis and Treatment Program (Trial Version 6)" (SHHCSATCM, 2020) guidance and recommendations, anti-viral therapy can be tested with alpha interferon (can be combined with ribavirin), Lopinavir/ritonavir (can be used in combination with ribavirin), chloroquine phosphate or abide. Cancer patients should suspend anti-tumor drug treatment while receiving anti-2019-nCoV virus treatment.

\section{Choice of a Treatment Plan for Cancer Patients During NCP Epidemic}

\section{a. Recommendations for Cancer Patients Receiving Medical Treatment}

The attending physician evaluates whether the treatment plan can be continued. For the administration route of drugs in the treatment plan by oral administration, it is recommended to maintain the original treatment plan. 
For example, targeted therapy for non-small cell lung cancer and endocrine therapy for breast cancer are oral medications. These drugs can be taken continuously when the patient's condition is stable. After the epidemic has passed, return to the hospital in time to evaluate the effect of drug treatment and adjust the treatment plan if necessary (SHHCSATCM, 2020).

For patients who cannot maintain the initial treatment or should be treated as soon as possible according to the condition, it is recommended to choose the plan based on the patient's treatment stage and the latest clinical guidelines and clinical diagnosis and treatment specifications. The choice of chemotherapy regimen is based on weighing the pros and cons, strictly grasping the indications, giving priority to oral chemotherapeutic drugs, preferentially using short-term infusion therapy and choosing a regimen with low adverse reactions and good patient tolerance and strictly calculating the drug dose. Unnecessary drug treatment should be avoided during treatment and the pharmacist can be asked to simplify the prescription. For chemotherapy drugs that may cause white blood cell decline, primary prevention should be taken. Long-term preparations are given priority if oxaliplatin combined with capecitabine (XELOX) has been started for patients with colon cancer who need adjuvant chemotherapy after surgery. The function of adjuvant chemotherapy can be retained due to the specific situation of the epidemic. It is recommended to adjust to the United States National Comprehensive Cancer the National Comprehensive Cancer Network (NCCN) guidelines (NCCN, 2020; Li et al., 2020) recommended capecitabine for single oral treatment. After the epidemic is over, the treatment plan is optimized according to the tumor's control.

b. Recommendations for Cancer Patients who have not Received Drug Treatment

The attending physician assesses whether the patient needs anti-neoplastic therapy. You can choose to use related support or auxiliary drug treatment according to the patient's actual situation for patients who do not require follow-up anti-tumor drug treatment. For patients who need anti-tumor drug treatment, it is recommended to refer to cancer patients receiving drug treatment in "3.1", weigh the pros and cons and choose a treatment plan. For example, for those who have not started adjuvant chemotherapy after colon cancer surgery and need to develop adjuvant chemotherapy, it is recommended to choose the capecitabine oral treatment recommended by the guidelines (NCCN, 2020).

\section{Management of Cancer Patients During the NCP Epidemic}

\section{a. Management of Administration Time}

\section{i) Fix the Medication Time, do not Adjust at Will}

For a long-term drug administration, the fixed administration time can facilitate patient self-management and improve medication compliance. If other treatment drugs are combined during the epidemic, it is recommended to take medicine as long as it requires treatment. If there is a time conflict, the patient is advised to consult a pharmacist.

\section{ii) Take Regularly to Avoid Missing}

If you miss the medication, it is recommended to determine whether the medication needs to be supplemented according to the missed medication time and the usage and dosage requirements in the drug instructions. The missed prescription cannot be refilled at will, easily cause medication accumulation (Zhu et al., 2020).

\section{b. Dose Administration}

Patients should be instructed not to change the dosage at random and the treatment plan should be strictly implemented. If the dosage needs to be adjusted, they should first communicate with the attending physician and adapt it according to the physician's recommendation. There is a risk of taking medication at will (Hassan et al., 2018).

\section{c. Management of Taking Method}

Do not change the way you take medicine at will. Suspended or controlled-release drugs can be broken or cut at will, which can cause the rapid and large-scale release of drugs, resulting in seriously adverse consequences.

\section{E. Management of Adverse Drug Reactions}

\section{a. Chemotherapy and targeted therapy drugs}

\section{i) Adverse Blood System Reactions}

Before treatment, patients should be evaluated for the risk of neutropenic fever based on the patient's tumor type, treatment plan and patient's risk factors and according to China's "Expert Consensus on the Diagnosis and Treatment of Neutropenia Caused by Tumor Chemotherapy (2019 Edition)" (CCSP, 2019; WHO, 2020a) The primary prevention and secondary prevention of Granulocyte Colony-Stimulating Factor (G-CSF) were carried out at different stages of chemotherapy (CCSP, 2019). In the case of an epidemic situation, it is recommended to choose longacting G-CSF to avoid patients frequently going to the hospital for injection treatment.

Increasing the accuracy of temperature measurements during treatment neutropenia may be accompanied by fever symptoms. The application of G-CSF may also cause fever, flu-like symptoms and other adverse reactions. Patients with fever should pay attention to distinguish with fever caused by the new coronavirus pneumonia. Patients should be instructed not to panic after the fever and communicate with the attending physician in time. 
After treatment, strengthen the monitoring of hematological indexes. Once abnormal hematological indexes occur, symptomatic treatment should be given in time according to the hematological toxicity guidelines or consensus of different tumor chemotherapy (CCSPCCCC, 2019a-b; Jun et al., 2016; Practice, 2019) to avoid serious bone marrow suppression. There may be insufficient blood preparation in medical institutions during the epidemic, so special attention should be paid to patients' bleeding risk and early treatment to reduce the need for blood transfusion.

\section{ii) Digestive System Adverse Reactions}

Digestive system reactions such as nausea, vomiting, or diarrhea are common adverse reactions of anti-tumor drugs. Before drug treatment, antiemetic drugs should be used prophylactically according to the assessment risk (Wenqi et al., 2019; Ghayvat et al., 2019; Mohd Su'ud et al., 2020). Before treatment, patients should be educated about a good lifestyle during treatment, such as eating less and eating more, controlling food intake, etc., to reduce nausea and vomiting. If the patient has diarrhea, attention should be paid to distinguish it from diarrhea caused by the new coronavirus infection. The symptomatic support treatment should strengthen the guidance antidiarrheal application drugs.

Long-term and combined application of anti-tumor drugs is likely to cause drug-induced liver injury (CSCO, 2016). Liver function and biochemical blood indicators should be closely monitored during treatment. Once abnormal liver function occurs, it should be promptly discontinued according to liver injury and clinical classification severity and given active drug treatment (Forni et al., 2017; Al-Shamsi et al., 2020).

\section{iii) Urinary System Adverse Reactions}

Traditional chemotherapy drugs and targeted drug immunotherapy may cause acute kidney injury in patients (Rosner et al., 2017; GPPA, 2020). It is recommended to strengthen patients' routine monitoring of serum creatinine and urine and combine with tumor type, stage, treatment plan and comorbidities.

\section{iv) Respiratory System Adverse Reactions}

Pulmonary toxicity caused by anti-tumor drugs can also cause symptoms such as coughing and dyspnea, which are more relevant to medicines. It should be distinguished from the symptoms of new coronavirus pneumonia.

\section{v) Other Adverse Reactions}

It is recommended to strengthen the patient's skincare education to reduce the skin reaction caused by chemotherapy and targeted therapy (CCPCCACA, 2019) to avoid the possibility of infection due to skin damage.

There is currently no effective drug to prevent neurotoxicity caused by chemotherapy. It is recommended to strengthen the education of patients on medication. If oxaliplatin is used, avoiding cold stimulation can prevent or reduce neurotoxicity.

If the patient has other intolerable adverse reactions, the patient should be informed to communicate with the attending physician to describe the clinical manifestations after taking medicine carefully. Then the physician will evaluate the patient's adverse reactions before proceeding accordingly.

\section{b. Immune Checkpoint Inhibitor}

The adverse reactions of immune checkpoint inhibitors are different from chemotherapy and targeted therapy. The more common ones are skin, endocrine, liver, gastrointestinal tract, lung, rheumatoid, skeletal muscle adverse reactions and infusion reactions. Adverse reactions in the heart, kidney, eyes, nerves, blood, etc., are rare (Xiaoxue et al., 2019; Thompson et al., 2019; Puzanov et al., 2017).

Attention should be paid to the following points in the management of immune checkpoint inhibitor adverse reactions: (1) Before treatment, the susceptibility of immunotherapy-related adverse reactions should be evaluated according to the risk factors of immune-related adverse reactions, the patient's particular circumstances and previous treatment conditions; (2) Closely monitor the emerging symptoms during treatment, identify them with other drugs or diseases themselves, assess the severity of their adverse reactions, determine whether glucocorticoids are required and the dosage form and dosage of glucocorticoids according to the grade of adverse reactions and Pay attention to monitor the harmful effects of glucocorticoids. (3) After treatment, the condition is stable and it is still necessary to monitor and evaluate the possible adverse reactions. Every three months in the first year, every six months after that (Manne et al., 2020).

\section{The Management of Drug Interactions}

Most cancer patients are elderly patients with underlying diseases. Also, they may be combined with other drugs during the epidemic. The risk of drug interactions increases, so attention should be paid. The recommendations are as follows: (1) Strengthen pharmacists' participation in the risk assessment of tumor drug interactions. For patients taking multiple drugs at the same time, the risk evaluation of drug interactions must be conducted by the pharmacist; (2) For medicines that have apparent or potential drug interaction risks, the physician should thoroughly discuss with the pharmacist, weigh the pros and cons and formulate a feasible treatment plan to ensure that the patient's treatment is completed during the epidemic. 


\section{F. Management of Daily Life of Cancer Patients During NCP Epidemic}

\section{a. Daily Protection}

Strengthen patient's and their families' education to prevent 2019-nCoV infection, strengthen personal protection, wear masks when going out, change clothes and wash hands after returning home, handle covers correctly and avoid environmental pollution in the home. Pay special attention to washing your hands frequently because hand hygiene is just as important as wearing a mask!

If family members and cancer patients have not been to the epidemic area and have no history of contact with 2019-nCoV-infected persons, no exceptional disinfection is required at home. Still, indoor ventilation should be paid attention to and care should be taken to keep the patients warm when opening windows. Patients and their families should try to isolate themselves as much as possible during the 2019-nCoV epidemic, minimize outings, reduce interpersonal communication and avoid door-to-door, dinner, or card games.

Suppose family members' occupation is inevitable to contact many people, such as doctors, civil servants, staff members, etc., who fight the virus or have colds and fever symptoms, even if the new coronavirus infection is temporarily ruled out. In that case, it is best not to contact patients with tumors. If the conditions do not allow you to have to live with the cancer patient and wear a mask at home and keep more than $1 \mathrm{~m}$ from the cancer patient, it is best to let them have their separate room. Pay attention to ventilation (open windows) in common areas (toilet, living room and dining room); clean frequently touched furniture daily with a disinfectant containing chlorine or peracetic acid; do not share tableware, towels and meals. If the family member finds that the tumor patient's condition is getting worse, seek medical treatment in time.

Family members with a history of travel in affected areas or suspected NCP patients should be isolated from cancer patients to avoid contact.

Patients should be educated to avoid fatigue and keep warm. Cancer patients should not be overworked; they should ensure adequate rest time, avoid long-distance travel and cause their immunity to decline, allowing the virus to take advantage of when winter and spring are in communication. It is essential to pay attention to cold and keep warm to avoid catching a cold.

Besides, it is currently in a critical period of epidemic prevention. If the tumor's condition has not deteriorated and the body is in good health, reduce the number of hospital visits to avoid infection. If re-examination is required, you can communicate with the attending physician, extend the time for returning to the hospital according to the actual situation and return to the hospital for re-examination after the epidemic has been alleviated.

\section{b. Concern about the Mental Health of Cancer Patients}

Cancer patients are often afraid of the disease and try to deny it. They are angry, easily grumpy, or cause depression. The patient's family may also have more psychological problems. During the NCP epidemic, social activities decreased and cancer patients were more likely to suffer from many psychological issues, such as anxiety, depression and fear that they could not be treated in time, resulting in headaches, poor appetite, insomnia, irritability and other symptoms and even amplified their discomfort. In particular, patients and their families who cannot be treated as scheduled because of the epidemic will think they are infected or think their condition is getting worse. Therefore, we should pay attention to psychological counseling's following aspects to patients and their families: (1) Learn to respond to the epidemic scientifically. During the epidemic, without excessive suspicion, choose mainstream media and authoritative organizations to receive information. (2) Communicate with friends and connect feelings through telephone, Internet, etc. Improve the quality of home life, such as doing housework, listening to music, reading books, etc. (3) Scientific arrange the work and rest time. While maintaining adequate sleep, we must maintain physical activity daily, effectively relieving emotions. (4) If more psychological severe problems occur, you can call for psychological consultation by telephone or go to a psychiatric clinic.

\section{c. Pay Attention to the Nutrition Management of Patients}

Cancer patients should pay attention to maintaining a diet with sufficient nutrition, a balanced diet, increasing the intake of high-quality protein and appropriately supplementing vitamins, minerals and dietary fiber.

If it is not necessary for the disease, the food should be separated as far as possible from wet and dry. Choose fewer porridges and soups with more water content and lower energy density and nutritional value. Patients with fever, dysphagia and other symptoms can make mixed food into a paste; if necessary, oral nutritional supplements and enteral nutritional preparations (exceptional medical food) are recommended to ensure that the daily additional energy intake is not less than $1674 \mathrm{~kJ}(1 \mathrm{kcal}=4.1858518 \mathrm{~kJ})$.

Variety of food types, sources, colors, meat and vegetables, to avoid a single; priority to ensure that based on staple food, protein, eat more fresh vegetables, fruits in moderation, drink plenty of water. The cooking methods are mostly steaming, boiling, stewing and braising. The food must be fully heated to avoid raw food.

Appropriately increase foods containing high-quality protein, such as fish, eggs, milk, skinless chicken and duck meat, beef and soy products; if intake is too small, protein powder can be selected for a supplement, but 
specific dosage and precautions should be consulted, Nutritionist. Do not listen to remedies, do not eat eggs or meat from wild animals, or do informal markets.

Due to the reduced intake, patients with tumours are prone to nutritional imbalances, especially inadequate intake of vitamins, minerals and dietary fiber. It is not recommended to consume many single vitamins alone and it is more recommended to obtain or take multivitamins from food (mainly fresh vegetables and fruits). Vitamin D supplementation is recommended for patients in isolation or outdoors. Foods rich in dietary fiber can improve constipation and maintain intestinal health. During the NCP epidemic, drink plenty of water to keep the respiratory tract and pharynx moist, preventing upper respiratory tract infections.

\section{Experimental Work}

Fluxicon Disco is one of the most popular Process Mining software to use data mining and discover the process's bottleneck. Fluxicon Disco contains the fastest process mining algorithms. Disco's revolutionary process mining technology allows you to construct beautiful visual maps from the process data in minutes, not weeks, according to its revolutionary process mining technology. Disco makes it fast and straightforward to improve efficiency, monitor deviations and experiment with new ideas. The most efficient log management and filtering framework to download the COVID-19. The Kaggle database has about 99 thousand records for cancer patients until December 2020. For the experiment, we chose the first 1,000 datasets from the Kaggle dataset Covid-19 cancer patient. There are fields patients_ID, treatment_date, background_diseases_diabetes, age, sex, smoking, or hypertension, new_treatment and regular_treatment. We open the Fluxicon Disco software dataset and import the case ID, activity, timestamp, resources and age from the dataset.

As mentioned in Table 1 performance analysis, there are 874 records used for an experiment in the thousands of datasets, 444 patient's dataset is applied to the new proposed algorithm and 430 patient's datasets are applied to a regular algorithm. Our experimental result shows the median duration of the new proposed algorithm 24 days is significantly less than the conventional treatment 41 days. The same as we discuss mean duration and duration range is significantly less time than traditional treatment. So as the results, our new proposed algorithms are more efficient rather than older regular algorithms Fig. 1.

As shown in Fig. 2. Performance analysis graphs are representing the new proposed treatment are suitable rather than the older treatment process. This graph shows the new proposed treatment frequency $42.16 \%$, like regular treatment frequency $41.17 \%$, but the proposed treatment performance is very high.

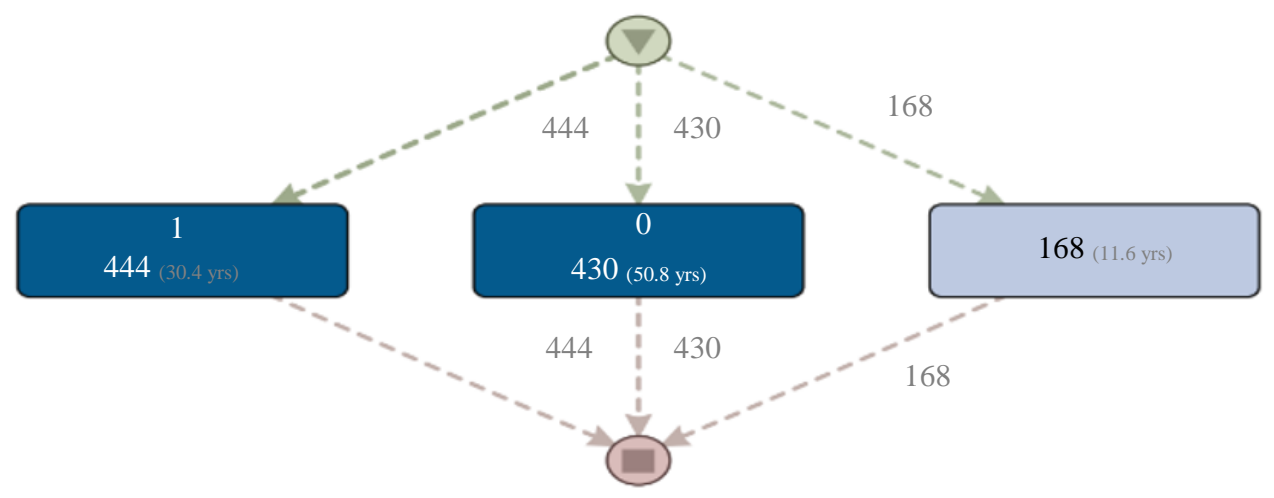

Fig. 1: Process map of Covid-19 Cancer patient dataset

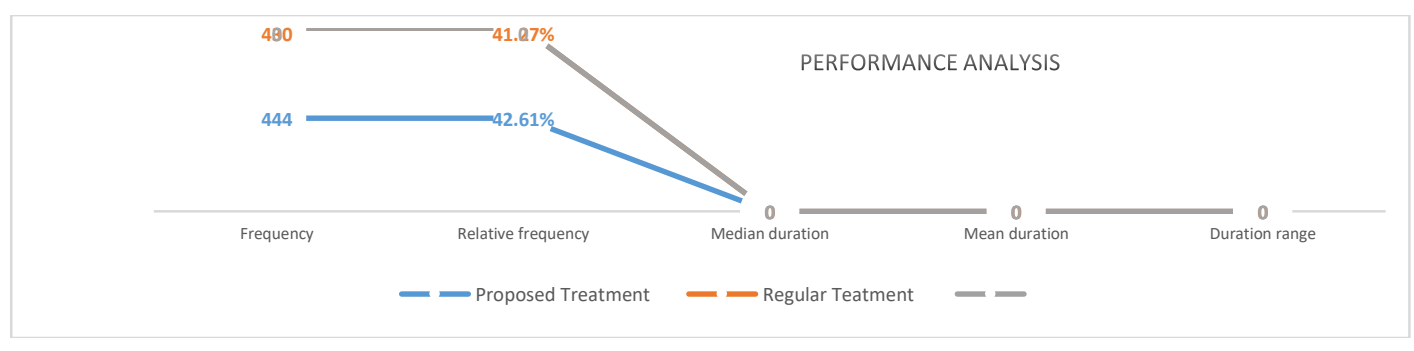

Fig. 2: Performance analysis graph 


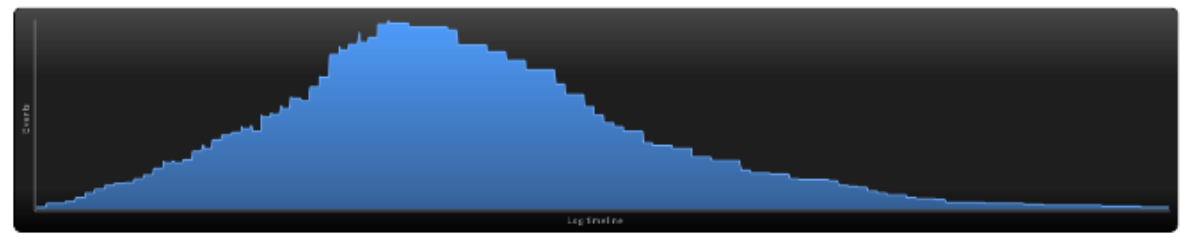

Fig. 3: Event over time

Table 1: Performance analysis with respect to time

\begin{tabular}{llllll}
\hline Treatment/time & Frequency & Relative frequency & Median duration & Mean duration & Duration range \\
\hline Proposed treatment & 444 & $42.61 \%$ & 24 days & 24 days, $23 \mathrm{~h}$ & 73 days \\
Regular treatment & 430 & $41.27 \%$ & 41 days & 43 days, $3 \mathrm{~h}$ & 102 days \\
\hline
\end{tabular}

\section{Results}

We discussed some of the existing problems associated with cancer patients' treatment during the COVID-19 pandemic in this review and offered some guidance and recommendations. This study aims to provide direction for cancer patients affected by Covid19 and provide the best care possible. This method is likely to refer to several pandemic infections. Figure 2 shows the proposed treatment takes less time to recover than regular medicine. Health care agencies will immediately begin preparing cancer treatment delivery during a pandemic. The limited but accumulative evidence indicates that cancer patients are at higher risk of COVID-19 infection than cancer-free individuals. During this COVID-19 pandemic, cancer patients' critical management approaches include consistent communication and education about hand hygiene, steps to prevent infections, high-risk exposure and COVID-19 signs and symptoms. In an infectious disease pandemic, consideration of risk and gain for aggressive intervention in the cancer community must be individualized. Care should be taken on a case-bycase basis for postponing elective surgery or chemotherapy for cancer patients with a reduced risk of progression. Minimizing the visits and elective admissions of outpatients will reduce the exposure and potential further transmission. As Fig. 3 shows, the bell curve graph that is normal distribution the curve shows the mean, mode and median of the data collection. Telemedicine can help patients reduce visits and infection risk in an infectious pandemic. More work is needed to clarify the virology and epidemiology of SARS CoV-2 in the cancer population.

\section{Acknowledgement}

My completion of this article could not have been accomplished without the support of my supervissor Dr. Sharinaz, Dr. Shafiza and my friends. To Sharinaz and Shafiza thank you for allowing me time away form you to research and write. Finally, to my caring, loving and supportive wife. It was a great comfort and relief to know that you were willing to provide management of our household activities while I completed my work. My heartfelt thanks.

\section{Author's Contributions}

Razi Ahmed: Conceived the ideas of experimental design of the study, performed experiments/data collection, data analysis and interpretation and writingoriginal draft preparation.

Shahrinaz Ismail: Conceived the ideas of experimental design of the study, writing review and editing and supervision.

Anwer Irshad Burney: Data analysis and interpretation and supervision.

Shafiza Mohd Shariff: Writing-review and editing and supervision.

Nawaf Waqas: Performed experiments/data collection.

\section{Ethics}

This research article has no harm to the researcher of the subject and no deceit laying in this article.

\section{References}

Al-Shamsi, H. O., Alhazzani, W., Alhuraiji, A., Coomes, E. A., Chemaly, R. F., Almuhanna, M., ... \& Xie, C. (2020). A practical approach to the management of cancer patients during the novel coronavirus disease 2019 (COVID-19) pandemic: an international collaborative group. The Oncologist, 25(6), e936. https://doi.org/10.1634/theoncologist.2020-0213

CCPCCACA. (2019). Practice Guidelines for Cancer Symptom Management in China-Skin Reaction [J]. Journal of Nurse Training, 2019, 34 (22): 2017-2024. https://doi.org/10.16821/j.cnki.hsjx.2019.22.001 
CCSP. (2019). Committee on Cancer Clinical Chemotherapy, China Cancer Society Professional Committee on Cancer Support Therapy. Expert consensus on the Diagnosis and Treatment of Neutropenia Caused by Cancer Chemotherapy (2019 Edition) [J]. Chinese Journal of Cancer Research, 2019, 46 (17): 876-882. doi.org/10.3969/j.issn.10008179.2019.17.913. Professional Committee of Cancer Clinical Chemotherapy of Chinese Anti-Cancer Association, Professional Committee of Cancer Supportive Therapy of Chinese Anti-Cancer Association. Consensus on the clinical diagnosis, treatment and prevention of chemotherapy-in-duced neutropenia in China (2019 edition) [J]. Chin J Clin Oncol, 2019, 46 (17): 876-882.

doi.org/10.3969/j.issn.1000 -8179.2019.17.913

CCSPCCCC. (2019a). China Cancer Society Professional Committee of Cancer Support Therapy. Chinese Cancer Chemotherapy-related Thrombocytopenia Expert Diagnosis and Treatment Consensus (2019 Edition) [J]. Chinese Journal of Cancer Research, 2019, 46 18): 923-929.

doi.org/10.3969/j.issn.1000-8179.2019.18.914

Professional Committee of Cancer Clinical Chemotherapy of Chinese Anti-Cancer Association, Professional Committee of Cancer Supportive Therapy of Chinese Anti-Cancer Association. Consensus on the clinical diagnosis, treatment and prevention of chemotherapy-in-duced thrombocytopenia in China (2019 version) [J]. Chin J Clin Oncol, 2019, 46 (18): 923-929. doi.org/10.3969/j.issn.1000- 8179.2019.18.914

CCSPCCCC. (2019b). China Cancer Society Professional Committee on Cancer Support Therapy. China Cancer Chemotherapy-Related Anemia Diagnosis and Treatment Expert Consensus (2019 Edition) [J]. China Cancer Society, 2019, 46 (17): 869-875. doi.org/10.3969/j.issn.1000-8179.2019.17.919.

Professional Committee of Cancer Clinical Chemotherapy of Chinese Anti-Cancer Association, Professional Committee of Cancer Supportive Therapy of Chinese Anti-Cancer Association. A consensus on the clinical diagnosis, treatment and prevention of cancer-and chemo-therapy-related anemia in China (2019 edition) [J]. Chin J Clin Oncol, 2019, $46 \quad$ (17): $\quad 869-875$. doi.org/10.3969/j.issn.1000 -8179.2019.17.919

Chen, Y. M., Perng, R. P., Chu, H., Tsai, C. M., \& WhangPeng, J. (2004). Impact of severe acute respiratory syndrome on the status of lung cancer chemotherapy patients and a correlation of the signs and symptoms. Lung Cancer, 45(1), 39-43.

doi.org/10.1016/j.lungcan.2004.01.002
CSCO. (2016). Hematology Branch of Chinese Medical Association, China Anti-Lymphoma Alliance. Expert consensus on the prevention and standardized treatment of drug-induced liver injury in hematological patients (2016 edition) [J]. Chinese Journal of Hematology, 2016, 37 (6): 441-452. https://doi.org/10.3760/cma.j.issn.0253-

2727.2016.06.001. Chinese Society of Clinical Oncology, Hematology Branch of Chinese Medical Association, China Anti-Lymphoma Alliance. Expert panel consensus statement on prevention and standardized treatment of drug-induced liver injury in patients with blood diseases (2016) [J]. Chin J Hematol, 2016, $37 \quad$ (6): 441-452. doi.org/10.3760/cma.j.issn.0253-2727.2016. 06.001

Favre, G., Pomar, L., Musso, D., \& Baud, D. (2020). 2019-nCoV epidemic: what about pregnancies?. Lancet (London, England), 395(10224), e40. https://www.ncbi.nlm.nih.gov/pmc/articles/PMC7 $133555 /$

Forni, D., Cagliani, R., Clerici, M., \& Sironi, M. (2017). Molecular evolution of human coronavirus genomes. Trends in microbiology, 25(1), 35-48. doi.org/10.1016/j.tim.2016.09.001

Ghayvat, H., Awais, M., Pandya, S., Ren, H., Akbarzadeh, S., Chandra Mukhopadhyay, S., ... \& Chen, W. (2019). Smart aging system: uncovering the hidden wellness parameter for well-being monitoring and anomaly detection. Sensors, 19(4), 766. https://doi.org/10.3390/s19040766

GPPA. (2020). Guidelines for the full-course pharmaceutical service of immune checkpoint inhibitors (2019 version) [EB/OL]. (2019-11-25) [2020-02-16]. Http://www.sinopharmacy. com.cn/notification/1792.html.Guangdong

Pharmaceutical Association. Guidelines for pharmaceutical care of immune checkpoint inhibitors in the whole process (2019 version) [EB/OL]. (2019-11-25) [2020-02-16]. http://www.sinopharmacy.com.cn/notification/17 92.html

Hassan, W., Jamil, N., Israr, M., \& Wajeeh, M. (2018, January). Study of gender preference of hepatitis virus in suspected patients from Islamabad and Karachi (Southern and northern parts of Pakistan). In 2018 15th International Bhurban Conference on Applied Sciences and Technology (IBCAST) (pp. 231-234). IEEE. doi.org/10.1109/IBCAST.2018.8312229

Huang, C., Wang, Y., Li, X., Ren, L., Zhao, J., Hu, Y., ... \& Cao, B. (2020). Clinical features of patients infected with 2019 novel coronavirus in Wuhan, China. The lancet, 395(10223), 497-506. /doi.org/10.1016/S0140-6736(20)30183-5 
Johnson, D. B., Balko, J. M., Compton, M. L., Chalkias, S., Gorham, J., Xu, Y., ... \& Moslehi, J. J. (2016). Fulminant myocarditis with combination immune checkpoint blockade. New England Journal of Medicine, 375(18), 1749-1755. https://www.nejm.org/doi/full/10.1056/NEJMoa1 609214

Jun, M., Jiejun, W., \& Li, Z. (2016). Tumor-related anemia clinical practice guidelines (2015-2016 edition) [J]. Chinese Journal of Practical Internal Medicine, 2016, 36 (S1): 1-21. MaJ, WangJJ, ZhangL, et al. Clinical guidelines on tumor associated anemia (Version 20152016) $[\mathrm{J}]$. Chinese Journal of Practical Internal Medicine, 2016, 36 (S1): 1-21.

Li, L., Zhang, Q., Wang, X., Zhang, J., Wang, T., Gao, T. L., ... \& Wang, F. Y. (2020). Characterizing the propagation of situational information in social media during covid-19 epidemic: A case study on weibo. IEEE Transactions on Computational Social Systems, 7(2), 556-562. https://doi.org/10.1109/tcss.2020.2980007.

Liang, W., Guan, W., Chen, R., Wang, W., Li, J., Xu, K., ... \& He, J. (2020). Cancer patients in SARS-CoV-2 infection: a nationwide analysis in China. The lancet oncology, 21(3), 335-337. https://doi.org/10.1016/S1470-2045(20)30096-6

Manne, R., Kantheti, S., \& Kantheti, S. (2020). Classification of Skin cancer using deep learning, ConvolutionalNeural Networks-Opportunities and vulnerabilities-A systematic Review. International Journal for Modern Trends in Science and Technology, ISSN, 2455-3778.

https://doi.org/10.46501/ijmtst061118

Mohd Su'ud, M., Ahmed, R., Faizan, M., Alam, M. M., \& Yafi, E. (2020). A Framework for Real-Time Healthcare System Performance in Developing Countries. https://doi.org/10.3844/jcssp.2020.1250.1257

Moslehi, J. J., Salem, J. E., Sosman, J. A., Lebrun-Vignes, B., \& Johnson, D. B. (2018). Increased reporting of fatal immune checkpoint inhibitor-associated myocarditis. The Lancet, 391(10124), 933. https://www.thelancet.com/journals/lancet/article/PI IS0140-6736(18)30533-6/supplemental

NCCN. (2020). NCCN clinical practice guidelines in Oncology: colon cancer (2020.V1) [EB/OL]. (2019-12-19) [2020-02-18].

Https://www.nccn.org/professionals/physician_gl s/default.aspx

Practice, B. B. (2019). Coronavirus disease 2019. World

Heal. Organ., pp, 2633, 2020.

https://doi.org/10.1001/jama.2020.2633
Puzanov, I., Diab, A., Abdallah, K., Bingham, C. 3. Brogdon, C., Dadu, R., ... \& Ernstoff, M. S. (2017). Managing toxicities associated with immune checkpoint inhibitors: consensus recommendations from the Society for Immunotherapy of Cancer (SITC) Toxicity Management Working Group. Journal for immunotherapy of cancer, 5(1), 1-28. https://doi.org/10.1186/s40425-017-0300-z

Raber-Durlacher, J. E., Barasch, A., Peterson, D. E., Lalla, R. V., Schubert, M. M., \& Fibbe, W. E. (2004). Oral complications and management considerations in patients treated with high-dose chemotherapy. Supportive cancer therapy, 1(4), 219-229. https://doi.org/10.3816/SCT.2004.n.014

Rosner, M. H., \& Perazella, M. A. (2017). Acute kidney injury in patients with cancer. New England Journal of Medicine, 376(18), 1770-1781. https://doi.org/10.1056/NEJMc1707248

SHHCSATCM. (2020). Diagnosis and treatment of new coronavirus pneumonia (trial sixth edition) [EB/OL]. (2020-02-18) [2020-02-19].

Http://www.gov.cn/zhengce/zhengceku/2020-

02/19/content_5480948.htm.NationalHealth

Commission of People's Republic of China, National Administration of traditional Chinese Medicine. Diagnosis and treatment of novel coronavirus pneumonia (revised version sixth) [ EB/OL]. (202002-18)

[2020-02-19].

Http://www.gov.cn/zhengce/zhengceku/202002/19/content_5480948.htm

SMSB. (2020). Notice of the Office of the National Medical Security Bureau on optimizing medical insurance handling services to promote the prevention and control of pneumonia outbreaks caused by new coronavirus infections [EB/OL]. -16]. Http://www.gov.cn/xinwen/2020-

02/04/content 5474456.htm. National Medical Security Administration. Notice of the Office of the National Medical Security Administration on optimizing medical security management services and promoting the prevention and control of pneumonia epidemic situation of novel coronavirus [EB/OL]. (2020-02-02) [2020-02-16]. http://www.gov.cn/xinwen/202002/04/content 5474456 .htm

Song, F., Shi, N., Shan, F., Zhang, Z., Shen, J., Lu, H., ... \& Shi, Y. (2020). Emerging 2019 novel coronavirus (2019-nCoV) pneumonia. Radiology, 295(1), 210-217. https://pubs.rsna.org/doi/full/10.1148/radiol.2020 200274

Thompson, J. A., Schneider, B. J., Brahmer, J., Andrews, S., Armand, P., Bhatia, S., ... \& Scavone, J. L. (2019). Management of immunotherapy-related toxicities, version 1.2019, NCCN clinical practice guidelines in oncology. Journal of the National Comprehensive Cancer Network, 17(3), 255-289. https://doi.org/10.6004/jnccn.2019.0013 
van Helvoort, T. (2014). 'Virus \& Cancer Studies'-Still fascinating after all these years (2014). Studies in History and Philosophy of Science Part C: Studies in History and Philosophy of Biological and Biomedical Sciences, 48, 258-259. https://doi.org/10.1016/j.shpsc.2014.09.001

Vlantis, A. C., Tsang, R. K., Wong, D. K., Woo, J. K., \& Van Hasselt, C. A. (2004). The impact of severe acute respiratory syndrome on otorhinolaryngological services at the Prince of Wales Hospital in Hong Kong. The Laryngoscope, 114(1), 171-174. https://onlinelibrary.wiley.com/doi/full/10.1097/000 05537-200401000-00032

Wenqi, J., Yi, B., \& Jifeng, F. (2019). Chinese Expert Consensus on the Prevention and Treatment of Cancer-related Nausea and Vomiting (2019 Edition) [J]. Chinese Medical Frontiers (Electronic Edition), 2019, 11 (11): 16-26. JiangWQ, BaY, FengJF, et al. Chinese experts' consensus on the prevention and treatment of nausea and vomiting related to anticancer drug treatment (2019 version) [J]. Chin J Front Med Sci (Electronic Version), 2019, 11 (11): 16-26.
WHO. (2020a). Coronavirus disease (COVID-19) outbreak [EB/OL]. (2020-01-30) [2020-02-16].

Xiaoxue, L., Jun, R., \& Xin, W. (2019). Review of studies on the toxicity management of immune checkpoint inhibitors [J]. China Medical Frontiers (electronic version), 2019, 11 (12): 27-31. https://doi.org/10.12037/YXQY.2019.12- 07.LiXX, RenJ, WangX, et al. Overview of research on toxicity management related to immune checkpoint inhibitors [J]. Chin J Front Med Sci (Electronic Version), 2019, 11 (12): $27-31$. https://doi.org/10.12037/YXQY.2019.12-07

Zhu, N., Zhang, D., Wang, W., Li, X., Yang, B., Song, J., ... \& Tan, W. (2020). A novel coronavirus from patients with pneumonia in China, 2019. New England Journal of medicine.

https://doi.org/10.1056/NEJMoa2001017 